\title{
Atomistic simulation of epitaxial interfaces and polytypes
}

\author{
G P DAS
}

Solid State Physics Division, Bhabha Atomic Research Centre, Mumbai 400085, India

\begin{abstract}
In this paper we discuss how ab initio local density electronic structure calculations can be used to investigate extended defects such as interfaces and polytypes. LMTO-supercell calculations have been performed to understand the nature of bonding in epitaxial metal/ceramic interfaces such as $\mathrm{Ag} / \mathrm{MgO}(001)$ and $\mathrm{Ti} / \mathrm{MgO}(001)$. Cohesive and electronic properties of hexagonal polytypes of diamond, with different stacking sequences, have been predicted for the first time and compared with the available experimental data. The relative stabilities of $4 \mathrm{H}, 6 \mathrm{H}$ and $8 \mathrm{H}$ diamond polytypes have been calculated using a generalized version of force theorem.
\end{abstract}

Keywords. Electronic structure; force theorem; metal/ceramic interface; diamond polytypes.

\section{Introduction}

Extended defects such as surfaces, interfaces, stacking faults, grain boundaries etc are encountered almost everywhere in materials science, and computer simulation is playing an increasingly crucial role in studying these defects. All the relevant parameters can be exactly specified and every minute details of the structure can be 'observed' in computer experiments, even when laboratory experiments fail to probe it. That is why computer simulation has now become indispensable to understand experimental data and to make predictions about results of experiments not yet feasible. There is a broad spectrum of length scales, right from nanometer to meter, which describe different systems and these are dictated by resolution of the phenomena under consideration. Table 1 summarizes the various kinds of modelling associated with these different length scales, the corresponding methodologies followed and the type of information extracted. Although the boundaries are not very well defined, this hierarchy of simulation falls broadly under the domain of solid state physicists, quantum chemists, physical metallurgists and materials engineers (Finnis 1993; Nguyen Manh et al 1995).

In this paper, I shall restrict myself to atomistic simulation of extended defects, which play crucial role in determining the cohesive and mechanical properties of metallic as well as insulating solids. Systematic efforts have been made to develop simple but accurate effective interatomic potentials (i.e. potential energy of a collection of atoms as a function of the atomic coordinates), which may describe the nature of bonding in metals, ceramics as well as their composites and interfaces. These empirical many-atom potentials (de Hosson 1980; Smith and Srolovitz 1992; Grimes et al 1996) though enable one to treat up to $\sim 10^{4}-10^{5}$ atoms, have inherent limitations. For example, the directional character of bonding in covalent solids cannot come out of these theories which are therefore not suitable for ceramics or semiconductors or even for intermetallics. The latter have unsaturated covalent bonds between different atomic species, making them candidates for high temperature high strength materials. The best way to overcome this problem is by bridging the gap between the atomistic (or micrometer) and electronic (or nanometer) length scales, and try to derive interatomic potentials based on quantum mechanical prediction. One such approach, used successfully on 
Table 1. Hierarchy of models for simulations in materials at various length scales.

\begin{tabular}{|c|c|c|c|c|}
\hline $\begin{array}{l}\text { Length } \\
\text { scale }\end{array}$ & Modelling & Methodology & Information & Discipline \\
\hline $\begin{array}{l}\mathrm{nm} \\
\leqslant 100 \text { atms }\end{array}$ & Electronic & $\begin{array}{l}\text { First principles HF } \\
\text { LDA electronic str. } \\
a b \text { initio mol. dyn. }\end{array}$ & $\begin{array}{l}\text { Electronic motion } \\
\text { Total En., 'Force' } \\
\text { Cluster Geometry }\end{array}$ & $\begin{array}{l}\text { Solid State/ } \\
\text { Quantum } \\
\text { Physics }\end{array}$ \\
\hline $\begin{array}{l}\mu \mathrm{m} \\
\leqslant 10^{3} \text { atms }\end{array}$ & Atomistic & $\begin{array}{l}\text { Empirical many-atom } \\
\text { potl. (TB, GPT, EMT) } \\
\text { Classical mol. dyn. }\end{array}$ & $\begin{array}{l}\text { Nature of chemical } \\
\text { bond, EPI, Interato- } \\
\text { mic force, Phase diagr. }\end{array}$ & $\begin{array}{l}\text { Materials Sci. } \\
\text { Quantum } \\
\text { Chemistry }\end{array}$ \\
\hline $\mathrm{mm}$ & Structural & $\begin{array}{l}\text { Monte Carlo, Pott's } \\
\text { model, Stochastic } \\
\text { Cellular Automata }\end{array}$ & $\begin{array}{l}\text { Microstructural } \\
\text { evolution, } \\
\text { Grain growth }\end{array}$ & $\begin{array}{l}\text { Physical } \\
\text { Metallurgy }\end{array}$ \\
\hline $\mathrm{m}$ & Continuum & $\begin{array}{l}\text { Finite difference, } \\
\text { Finite element }\end{array}$ & $\begin{array}{l}\text { Elastic and plastic } \\
\text { response under } \\
\text { applied load }\end{array}$ & $\begin{array}{l}\text { Materials } \\
\text { Engineering }\end{array}$ \\
\hline
\end{tabular}

intermetallics, is the so-called tight-binding bond order potential model (Pettifor 1989; Horsfield 1996), where the angular dependence of the many-atom potential is explicitly taken into account. Then there are other models which have been proposed to explain the ionic bonding in oxide ceramics (Lambrecht and Segal 1989). However, none of the abovementioned methods are universally applicable to all the different types of bonding and one cannot expect something like the 'best potential function'.

A microscopic understanding of the problems such as bonding in metal/ceramic interface necessitates a higher level of sophistication - which is provided by $a b$ initio quantum mechanical approaches. Obviously there has to be a trade-off between the level of sophistication (or equivalently robustness or reliability) of a particular method and the number of non-equivalent atoms it can handle (or equivalently speed) (Vitek and Srolovitz 1989; Smith and Srolovitz 1992). For example, the most accurate first principles local density electronic structure or molecular dynamics (Car-Parrinello) methods can be used today for $\sim 100$ atom cluster or supercell, while for handling defects containing $\sim 1000$ atoms one has to take recourse to some tight-binding schemes (Pettifor and Cottrell 1992).

\section{Methodology of calculation}

I shall discuss in this paper the applications of local density functional approach to epitaxial interfaces and polytypes - both of which are classified by the materials scientists as extended defects. The various computational schemes for atomistic simulations, based on density functional approach, have been amply discussed in the literature (see e.g. a recent overview by Freeman and Wimmer 1995). To handle interfaces or polytypes, the first crucial step is to create a suitable geometrical model of the system which in this case is a supercell with periodic boundary condition along the stacking direction. Thus the system is reduced to an effective one dimensional problem. The next step is to choose an appropriate one electron band structure method for calculating the ground state total (internal) energy $E[\rho]$ at $0^{\circ} \mathrm{K}$ of a system of electrons interacting with each other in presence of an external potential. By differentiating the total energy w.r.t. the position coordinate of a given atom, we can get the quantum 
mechanical 'force' acting on it due to all the other atoms (Nielsen and Martin 1985). The minimum energy corresponds to zero force on all atoms. That way, one can obtain the equilibrium geometry of an epitaxial interface or a polytype, and also its energetics.

We have used LDA-based tight-binding linear muffin-tin orbital (TBLMTO) method (Andersen and Jepsen 1984, 1987) in the atomic sphere approximation (ASA) (Andersen 1975). The method is fast, efficient and ideally suited for large supercells with relatively low symmetry (Das 1992, 1994). Due to shape approximation in potential and charge density, the ASA total energy has some error which is minimized while determining the energy differences in close-packed solids. Furthermore, one can use the so-called Andersen force theorem (Andersen et al 1979; Mackintosh and Andersen 1980) to estimate the minute energy differences between various structures related by first order distortion of the lattice and having more or less the same packing fraction. The force theorem (FT) essentially takes advantage of the stationary property of the total ground state energy w.r.t. variation of the ground state charge density $\rho_{\mathrm{gs}}(\mathbf{r})$ i.e.

$$
E\left[\rho_{\mathrm{gs}}(\mathbf{r})+\delta \rho(\mathbf{r})\right]=E\left[\rho_{\mathrm{gs}}(\mathbf{r})\right]+O(\delta \rho(\mathbf{r}))^{2} .
$$

Thus, if a system A having self-consistent ground state charge density $\rho_{A}^{\text {sc }}(\mathbf{r})$ and ground state energy $E_{\mathrm{A}}\left[\rho_{\mathrm{A}}^{\mathrm{SC}}(\mathbf{r})\right]$, is deformed by rigid displacement of atoms to another system $B$, whose ground state energy $E_{\mathrm{B}}$ is calculated by the so-called frozenpotential (FP) prescription (Andersen et al 1979; Mackintosh and Andersen 1980) (i.e. by placing the potential $v_{\mathrm{A}}(\mathbf{r})$ of system $\mathrm{A}$ at the atomic positions of the system $\mathrm{B}$ ), then change in the total energy (which is proportional to 'force') can be written as a restricted variation:

$$
\delta E=E_{\mathrm{B}}\left[\rho_{\mathrm{B}}^{\mathrm{FP}}(\mathbf{r})\right]-E_{\mathrm{A}}\left[\rho_{\mathrm{A}}^{\mathrm{SC}}(\mathbf{r})\right]=\delta\left(\sum_{i}^{o c c} E_{i}\right)+\delta E_{\mathrm{M}} .
$$

Here $\delta\left(\Sigma_{i}^{o c c} E_{i}\right)$ is the change in the occupied eigenvalues (one electron band energies) of the two systems and $\delta E_{\mathrm{M}}$ the change in electrostatic Madelung energies as a result of displacing the atoms from system $A$ to system $B$. The practical recipe for this restricted variation becomes particularly simple within the ASA, since it separates the potential dependent part from the crystal structure dependent part of the energy band problem. The use of frozen (i.e. not self-consistently relaxed) potential to generate $\rho_{\mathrm{B}}^{\mathrm{FP}}$ ensures that the chemical shifts in the tightly bound core electron energies drop out of the above equation; also the double counting electrostatic (Hartree) terms and the exchange correlation terms cancel exactly (Andersen et al 1979; Mackintosh and Andersen 1980; Heine 1980). Ultimately what survives is only the sum of the one electron energies of the valence or band electrons, which is entirely quantum mechanical in origin. The second term $\delta E_{M}$ in (2) is a correction term which vanishes to the first order within ASA, since the electrostatic Madelung energy is approximated by that of the neutral Wigner-Seitz (WS) sphere, the neutrality remaining unaffected by the change in structure.

The force relation (2) is now widely recognized as an extremely accurate approximation and had been applied with remarkable success to delineate the crystal structure sequence with increasing atomic number across the transition metal series (Skriver 1985). Recently, force theorem has also been used to determine stacking fault energies in close packed fcc metals (Crampin et al 1990; Ernst et al 1992). 


\section{Metal/ceramic interfaces}

A number of semi-empirical approaches, like the EMT, embedded atom model, glue model etc have been deployed with varying degree of success in order to extract the effective interatomic potentials in a metal or in an insulator. But when it comes to a heterophase boundary between say a metal and an insulator, no suitable interatomic potential can be cooked which might be matched across the interface. This is where one needs to go to a rigorous first principles electronic structure calculation, which takes care of the charge rearrangements across the interface self-consistently. The nature of bonding across the boundary between an oxide ceramic (such as $\mathrm{MgO}$ ) and a transition/noble metal (such as Ti or Ag) have been successfully derived from self-consistent LMTO calculations and also it has been possible to quantify the work of adhesion surface energy, interface energy etc (Blöchl et al 1990; Schönberger et al 1992). Experimentally much less is known about the structures and adhesions of interfaces than about surface structures and energies. The bonding between an oxide ceramic and a d-band metal is less well understood than that with a free electron like metal. In case of $\mathrm{Ti} / \mathrm{MgO}(100)$ and $\mathrm{Ag} / \mathrm{MgO}(100)$, the lattice misfits are $2 \%$ and $3 \%$ respectively, and the misfit dislocations at interfaces between weakly bonded partners are likely to be strongly delocalized as observed in TEM studies (Trampert et al 1992). In order to eliminate the misfit in supercell calculations, the metal lattice is expanded parallel to the interface (with a concomitant contraction perpendicular to the interface), leaving the stiffer $\mathrm{MgO}$ crystal undeformed. The supercell chosen for our calculation (Blöchl et al 1990) has tetragonal symmetry and contains two identical interfaces separated by 10 atomic layers. This thickness is sufficient to make the material at the centre of each slab virtually identical to the bulk material.

Bulk $\mathrm{MgO}$ is an ionic insulator with large direct gap of $7.8 \mathrm{eV}$, and its valence band structure is predominantly $\mathrm{O}-2 \mathrm{p}$ character with a width of $2.5 \mathrm{eV}$. On the other hand, the band structure of $\mathrm{Ag}$ is characterized by a broad free-electron like s-band and a nearly full d-band, while that of Ti has a less than half-filled d-band. From our self-consistent supercell calculations, we have extracted the layer-projected density of states (LP-DOS), which reveal both the abovementioned features in the respective bulk-like layers. In the interface layers, however, the LP-DOS is strongly perturbed due to hybridization between TM-s states and the $\mathrm{O}-p$ and $\mathrm{Mg}-s, p$ states. Some 'virtual gap states' appear in the band gap close to an interface, which is well known for metal-semiconductor interfaces (Das $1992,1994)$. There is also charge transfer from the $\mathrm{MgO}$ valence band to the $\mathrm{TM}$, which prefers to occupy the oxygen-top position, as suggested by our total energy calculations (Blöchl et al 1990). In order to estimate the interface distance, force constant, adhesion etc more accurate full potential LMTO calculation had been performed (Schönberger et al 1992). The work of adhesion for $\mathrm{Ag} / \mathrm{MgO}(001)$ was found to be $0.84 \mathrm{eV} /$ interface, while that for $\mathrm{Ti} / \mathrm{MgO}(001)$ it was $1.22 \mathrm{eV} /$ interface, thereby suggesting that $\mathrm{Ti}$ bonds are stronger than $\mathrm{Ag}$ to the $\mathrm{MgO}$ surface. The bonding between $\mathrm{Ag}$ and $\mathrm{O}$ is weak and predominantly ionic, while that between $\mathrm{Ti}$ and $\mathrm{O}$ is predominantly covalent. This fact is supported by the observation that the adhesion correlates with the free energy of oxide formation for the d-band metals, both increasing in the order $\mathrm{Ag} \rightarrow \mathrm{Ni} \rightarrow \mathrm{Fe} \rightarrow \mathrm{Ti}$.

\section{Diamond polytypes}

Diamond is a classic example of a crystalline insulating material available in nature with a unique combination of structural, thermal, optical and electronic properties. During the 
last decade or so, considerable effort has been made to prepare synthetic diamond and diamond-like-carbon (DLC) films using low pressure epitaxial growth techniques such as chemical vapour deposition. Significant amount of literature exists on the synthesis, characterization and applications of diamond films (Brown 1990). From fundamental point of view also, this diamond family is interesting because of manifestation of hexagonal polytypes having the same $s p^{3}$ bonding as normal cubic diamond but with different stacking sequence (Spear et al 1990). While lonsdalite (or $2 \mathrm{H}$ ) diamond was discovered way back in 1967 (Bundy and Kasper 1967) using high pressure methods, higher polytypes viz. $4 \mathrm{H}$ and $6 \mathrm{H}$ diamond have been synthesized only recently (Frenklach et al 1989) e.g. using laser-indisced reactive quenching at a liquid-solid interface (Sharma et al 1993). However, only a few attempts have been made to investigate the electronic and cohesive properties and the nature of bonding in diamond polytypes (Salehpour and Satpathy 1990; Sharma et al 1996; Salunke et al 1997).

Here we present the results of our self-consistent LDA supercell calculation on $4 \mathrm{H}, 6 \mathrm{H}$ and $8 \mathrm{H}$ diamond polytypes, which may be looked upon as ordered stackings (along c-direction) of cubic (k) and hexagonal (h) units (Spear et al 1990; Salunke et al 1997). The first part of table 2 summarizes the essential structural information on all the diamond polytypes including those of the parent structures viz. $3 \mathrm{C}$ (pure $\mathbf{k}$ ) and $2 \mathrm{H}$ (pure h). For each structure, we have calculated the total ground state energy (at $T=0$ ) as a function of the unit cell volume. These energy-vs-volume parabolae show maximum stability for $3 \mathrm{C}$ as expected, and minimum stability for $2 \mathrm{H}$ diamond, whose cohesive energy is about $1.2 \mathrm{eV}$ lower (see table 2). Interestingly, the parabolae for $4 \mathrm{H}$, $6 \mathrm{H}$, and $8 \mathrm{H}$ polytypes lie intermediate between $3 \mathrm{C}$ and $2 \mathrm{H}$, and these metastable structures seem to compete with each other with extremely close ground state energies. In order to understand their relative stability, we have attempted to use a generalized version of Andersen force theorem (Andersen et al 1979; Mackintosh and Andersen 1980 ) in conjunction with LMTO-ASA. In our approach, we first extract the selfconsistent (SC) potentials and total ground state energies of $3 \mathrm{C}$ (pure k) and $2 \mathrm{H}$ (pure h) structures. Each $\mathbf{k}$ - or h-unit consists of two carbon atoms (one double layer) and two

Table 2. Results of self-consistent LMTO calculation of total energies and forces in various diamond polytypes (bond length $=1.546 \AA$ for all). Space group is $D_{6 h}^{4}$ for $n H$ polytypes.

\begin{tabular}{|c|c|c|c|c|c|}
\hline Polytype & $3 \mathrm{C}$ & $2 \mathrm{H}$ & $4 \mathrm{H}$ & $6 \mathrm{H}$ & $8 \mathrm{H}$ \\
\hline Stacking seq. & $\mathrm{ABC}$ & $\mathrm{AB}$ & $\mathrm{ABCB}$ & $\mathrm{ABCACB}$ & $\mathrm{ABCBACBC}$ \\
\hline Hägg notation & + & - & ++-- & $+++\cdots-$ & ++++--- \\
\hline Jagodzinski notation & $\mathbf{k}$ & h & hk & hkk & hkkk \\
\hline cubicity $(\alpha)$ & 1.000 & 0.000 & 0.500 & 0.667 & 0.750 \\
\hline $\mathbf{c} / \mathbf{a}$ & $2 \cdot 449$ & 1.633 & $3 \cdot 266$ & $4-899$ & 6.532 \\
\hline $\begin{array}{l}\text { Equilibr. lattice } \\
\text { constant }(\AA)\end{array}$ & $2 \cdot 592$ & $2 \cdot 673$ & $2 \cdot 669$ & $2 \cdot 666$ & $2 \cdot 655$ \\
\hline $\begin{array}{l}\text { Equilibr. bond } \\
\text { length }(\AA)\end{array}$ & $1 \cdot 588$ & 1.637 & 1.635 & 1.633 & $1 \cdot 626$ \\
\hline Bulk mod. (MBar) & $5 \cdot 061$ & $4 \cdot 451$ & $4 \cdot 277$ & $4 \cdot 288$ & $3 \cdot 343$ \\
\hline Coh. energy $(\mathrm{GV} / \mathrm{at})$ & $8 \cdot 690$ & $7 \cdot 450$ & 7.596 & $7 \cdot 616$ & $7 \cdot 598$ \\
\hline 'Force' (eV/at) & - & - & 0.148 & 0.235 & 0.306 \\
\hline Direct gap (eV) & $6 \cdot 496$ & $5 \cdot 003$ & 5.809 & 5.906 & 6.060 \\
\hline Indirect gap (eV) & $\begin{array}{c}5 \cdot 722 \\
(\Gamma \rightarrow M)\end{array}$ & $\begin{array}{c}2 \cdot 977 \\
(\Gamma \rightarrow K)\end{array}$ & $\begin{array}{c}5 \cdot 326 \\
(\Gamma \rightarrow M)\end{array}$ & $\begin{array}{c}5 \cdot 355 \\
(\Gamma \rightarrow M)\end{array}$ & $\begin{array}{c}5 \cdot 460 \\
(\Gamma \rightarrow M)\end{array}$ \\
\hline
\end{tabular}


empty spheres whose positions and radii are chosen such that the entire unit is close-packed (with reasonable sphere overlap) as well as charge neutral. Then we utilize the concept of transferability of these SC potentials to an hybrid $n \mathrm{H}$-polytype $(n=4,6,8)$ structure, and estimate its total ground state energy as $E_{\text {est }}=\alpha E_{\mathrm{k}}^{\mathrm{SC}}+$ $(1-\alpha) E_{\mathrm{h}}^{\mathrm{SC}}$, where the cubicity fraction $\alpha=n_{\mathrm{k}} /\left(n_{\mathrm{h}}+n_{\mathrm{k}}\right)$ increases from $1 / 2$ to $2 / 3$ to $3 / 4$ as one goes from $4 \mathrm{H}$ to $6 \mathrm{H}$ to $8 \mathrm{H}$ polytype. This $E_{\text {est }}$ should differ from the so-called frozen potential (FP) (Mackintosh and Andersen 1980) energy $E_{\mathrm{nH}}^{\mathrm{FP}}$ because of the environmental effect arising out of the way the two building blocks $h$ and $k$ are arranged. $\delta E=E_{\mathrm{nH}}^{\mathrm{FP}}-E_{\mathrm{est}}$ is a measure of the 'force' generated within the structure, which, according to Force Theorem, is controlled mainly by the one electron band structure energy. We find a systematic increase in $\delta E$ from $4 \mathrm{H}$ to $6 \mathrm{H}$ to $8 \mathrm{H}$, implying lesser stability for higher- $n$ polytypes. In support of our conclusion, it was shown long back by Schneer (1995) and Verma and Krishna (1966), using general thermodynamic arguments on $\mathrm{SiC}$ polytypes, that the interaction energy increases as a function of cubicity $(\alpha)$ and that the $4 \mathrm{H}$ structure is the most stable, followed by $6 \mathrm{H}$ and $8 \mathrm{H}$ polytypes. This interaction energy of an assembly of $h$ and $k$ units has been defined (Schneer 1955) as

$$
E=(2 \alpha-1) N \varepsilon,
$$

where $\varepsilon$ is the difference between the interaction energies of two like (hh and $\mathbf{k k}$ ) and unlike (hk) adjacent layers, and $N$ the total number of bilayers in the crystal. The most stable arrangement is expected to be the one which minimizes $E$. It was shown for higher polytypes of $\mathrm{SiC}$ that $E$ is minimum for $4 \mathrm{H}(\alpha=1 / 2)$ and progressively increases for $6 \mathrm{H}(\alpha=2 / 3)$ and $8 \mathrm{H}(\alpha=3 / 4)$. It is indeed encouraging to see that the trend in the forces (table 2 ) obtained by us via the force theorem route matches with this result. It would be interesting to apply the force theorem to other systems showing polytypism.

Coming back to the cohesive properties of the various polytypes, we have calculated the equilibrium lattice parameters and bulk moduli from the first and second derivatives of the total energy with respect to the cell volume. Our calculated in-plane lattice parameters (or equivalently bond lengths) systematically decrease, while going from $2 \mathrm{H}$ to $8 \mathrm{H}$ reaching a minimum for $3 \mathrm{C}$, which is rather close to the experimental value $(2 \cdot 522 \AA)$. Note that the cell parameter along the stacking direction can be taken as integral multiples of the unit layer separation (see table 2). The bulk moduli for the $4 \mathrm{H}, 6 \mathrm{H}$ and $8 \mathrm{H}$ polytypes are more or less same ( $\sim 4.3 \mathrm{MBar}$ ), lower than the corresponding values for $2 \mathrm{H}$ diamond (4.45 $\mathrm{MBar}$ ) and $3 \mathrm{C}$ diamond (5.06 MBar). Thus, if the higher diamond polytype films can be deposited on technologically important substrates, these should show significant hardness, albeit less than that of cubic diamond. The features of the electronic structures are revealed by the one electronic densities of states (DOS) which show wide difference between $3 \mathrm{C}$ and $2 \mathrm{H}$ structures, e.g. the usual three-humped structure of the occupied part of the DOS in $3 \mathrm{C}$ diamond changes to a step-like multi-peaked curve without any pseudogap in case of $2 \mathrm{H}$. The band gap remains indirect in both, but with different characters as well as magnitudes, as shown in table 2. For the higher $n \mathrm{H}$ polytypes, the band gaps remain more or less same. The interband transitions obtained for $3 \mathrm{C}$ and $4 \mathrm{H}$ polytype from the 1-projected DOS's have been found to match with the XPS energy loss data (Lurie and Wilson 1977; Sharma et al 1996). 
To conclude, we have illustrated in this paper, how the LDA-based TBLMTO-ASA electronic structure method can be deployed to investigate (a) the nature of chemical bonding in metal/ceramic interfaces and (b) the relative stabilities of diamond polytypes.

\section{Acknowledgements}

I would like to express my sincere thanks to P Ayyub, P Blöchl, H G Salunke, A K Sharma and U Schönberger, for fruitful collaborations and many helpful discussions.

\section{References}

Andersen O K 1975 Phys. Rev. B12 3060

Andersen O K and Jepsen O 1984 Phys. Rev. Lett. 532571

Andersen O K, Skriver H L, Nohl H and Johanson B 1979 Pure Appl. Chem. 5293

Andersen O K, Jepsen O and Sob M 1987 in Electronic band structure and its applications (ed.) M Yussouff (Berlin: Springer Verlag) p.1

Blöchl P, Das G P, Fischmeister H and Schönberger U 1990 in Metal-ceramic interfaces (eds) M Rühle et al (Oxford: Pergamon Press) p. 35

Brown W L (ed.) 1990 See for e.g. the collection of articles in the special issue on "Diamond like Carbon"; $J$. Mater. Res. 5 2273-2609

Bundy F P and Kasper J S 1967 J. Chem. Phys. 463437

Crampin S, Hampel K, Vvedensky D D and MacLaren J M 1990 J. Mater. Res. 52107

Das G P 1992 Pramana-J. Phys. 38545

Das G P 1994 in Lectures on methods of electronic structure calculations (eds) V Kumar, O K Andersen and A Mookerjee (Singapore: World Scientific) p. 279

de Hosson J Th M 1980 in Interatomic potentials and crystalline defects (ed.) J K Lee (Pittsburgh: The Metallurgical Society of AIME) p. 3

Ernst F, Finnis M W, Hofmann D, Muschik T, Schönberger U, Wolf U and Methfessel M 1992 Phys. Rev. Lett. 69620

Finnis M 1993 Phys. World 637

Freeman A J and Wimmer E 1995 Annu. Rev. Mater. Sci. 257

Frenklach M, Krematick R, Huang D, Howard W, Spear K E, Phelps A W and Koba R 1989 J. Appl. Phys. 66395

Grimes R W, Harker A H and Lidiard A B (eds) 1996 Interatomic potentials; Philos. Mag. 73 1-199

Heine V 1980 in Solid state physics (eds) H Ehrenreich, F Seitz and D Turnbull (New York: Academic Press) Vol. 35, p. 1 (A detailed argument and derivation of force theorem is given in pp 114-120)

Horsfield A 1996 Philos. Mag. B73 85

Lambrecht W R L and Segal B 1989 in Interfaces in metal-ceramic composites-Part II (eds) R Y Yin et al (Warrendale: The Minerals, Metals and Materials Society) p. 319

Lurie P G and Wilson J M 1977 Surf. Sci. 65476

Mackintosh A R and Andersen O K 1980 in Electrons at Fermi surface (ed.) M Springford (New York: Cambridge Univ. Press) p. 149 (Force theorem is discussed on pp 187-193)

Nielsen O H and Martin R M 1985 Phys. Rev. B32 3780

Nguyen Manh D, Bratkovsky A M and Pettifor D G 1995 Philos. Trans. R. Soc. London A351 529

Pettifor D G 1989 Phys. Rev. Lett. 632480

Pettifor D G and Cottrell A H (eds) 1992 Electron theory in alloy design (London: The Institute of Materials); see articles by D G Pettifor and A T Paxton

Salehpour M R and Satpathy S 1990 Phys. Rev. B41 3048

Salunke H G, Sharma A K, Das G P and Ayyub P 1997 (to be published)

Schneer C J 1955 Acta Cryst. 8279

Schönberger U, Andersen O K and Methfessel M 1992 Acta Metall. Mater. 40 S1 
Sharma A K et al 1993 Mater. Lett. 1742

Sharma A K, Salunke H G, Das G P, Ayyub P and Multani M S 1996 J. Phys.: Condens. Matter 85801

Skriver H L 1985 Phys. Rev. B31 1909

Spear K E, Phelps A W and White W B 1990 J. Mater. Res. 52277

Smith J R and Srolovitz D J 1992 Modelling Simul. Mater. Sci. Engg. 1101

Trampert A, Ernst F, Flynn C P, Fischmeister H F and Rühle M 1992 Acta Metall. Mater. 40 S227

Verma A R and Krishna P 1966 Polymorphism and polytypism in crystals (New York: John Wiley); (see the derivation given in pp 273-276)

Vitek V and Srolovitz D J (eds) 1989 Atomistic simulation of materials (New York: Plenum) 